\title{
STAT3 single nucleotide polymorphism rs4796793 SNP does not correlate with response to adjuvant IFN $\alpha$ therapy in stage III melanoma patients
}

\author{
David Schrama ${ }^{1}$, Selma Ugurel ${ }^{1,2}$, Antje Sucker ${ }^{2}$, Cathrin Ritter ${ }^{3,4}$, Marc Zapatka $^{5}$, Dirk Schadendorf ${ }^{2}$ and \\ Jürgen Christian Becker ${ }^{2,3,4 *}$
}

${ }^{1}$ Department of Dermatology, University Hospital Würzburg, Würzburg, Germany

${ }^{2}$ Department of Dermatology, University Hospital Essen, Essen, Germany

${ }^{3}$ Department of Dermatology, Medical University of Graz, Graz, Austria

${ }^{4}$ Department of Translational Skin Cancer Research, German Cancer Research Center, Heidelberg, Germany

${ }^{5}$ Department of Molecular Genetics, German Cancer Research Center, Heidelberg, Germany

\section{Edited by:}

Willeke Blokx, Radboud University

Medical Center, Netherlands

Reviewed by:

Remco Van Doorn, Leiden University Medical Center, Netherlands

Adriana Van Marion, VieCuri Medical

Centre, Netherlands

*Correspondence:

David Schrama, Department of Dermatology, University Hospital of Wuerzburg, Josef-Schneider-Str. 2, 97080 Wuerzburg, Germany e-mail:schrama_d@ukw.de;

Jürgen Christian Becker, Department of Translational Skin Cancer Research (TSCR), German Cancer Research Forum (DKTK), Medical University of

Essen, Universitätsstr. 1, 45147

Essen, Germany

e-mail: j.becker@dkfz.de
Interferon alpha (IFN $\alpha$ ) is approved for adjuvant treatment of stage III melanoma in Europe and the US. Its clinical efficacy, however, is restricted to a subpopulation of patients while side effects occur in most of treated patients. Thus, the identification of predictive biomarkers would be highly beneficial to improve the benefit to risk ratio. In this regard, STAT3 is important for signaling of the IFN $\alpha$ receptor. Moreover, the STAT3 single-nucleotide polymorphism (SNP) rs4796793 has recently been reported to be associated with IFNa sensitivity in metastatic renal cell carcinoma. To translate this notion to melanoma, we scrutinized the impact of rs4796793 functionally and clinically in this cancer. Interestingly, melanoma cells carrying the minor allele of rs4796793 were the most sensitive to IFN $\alpha$ in vitro. However, we did not detect a correlation between SNP genotype and STAT3 mRNA expression for either melanoma cells or for peripheral blood lymphocytes. Next, we analyzed the impact of rs4796793 on the clinical outcome of 259 stage III melanoma patients of which one-third had received adjuvant IFN $\alpha$ treatment. These analyses did not reveal a significant association between the STAT3 rs4796793 SNP and patients' progression free or overall survival when IFN $\alpha$ treated and untreated patients were compared. In conclusion, STAT3 rs4796793 SNP is no predictive marker for the efficacy of adjuvant IFN $\alpha$ treatment in melanoma patients.

Keywords: melanoma, interferon, predictive marker, STAT3, single nucleotide polymorphism

\section{INTRODUCTION}

Malignant melanoma is an aggressive skin cancer originating from melanocytes. Due to the continuously raising incidence of melanoma among the Caucasian population, it represents an increasing health problem. Indeed, in the US, melanoma is one of the few common cancers with increasing incidence rates over the last decade, i.e., a $2.4 \%$ increase per year among white women and $2.1 \%$ among white men during $1999-2009$ (1). A total of 68,130 new cases of melanoma, and 8,700 patients dying from melanoma had been predicted for 2010 in the US (2). Moreover, the economic burden of this disease is also reflected by the recent report that an individual in the US loses on average 20.4 years of their potential lifetime as a result of melanoma mortality compared with 16.6 years for all other malignant cancers (3).

The prognosis of a melanoma patient is largely dependent on the stage of disease. While early-stage melanoma can be cured in most cases by surgical excision of the tumor, already for patients with loco-regional disease beyond the primary tumor the

Abbreviations: IFN $\alpha$, interferon alpha; PBL, peripheral blood lymphocytes; STAT3, signal transducer and activator of transcription 3; SNP, single-nucleotide polymorphism. prognosis is highly impaired. Among patients with nodal metastases, the 5 -year survival rates were 78,59 , and $40 \%$ for patients with stage IIIA, IIIB, and IIIC melanoma, respectively. The subgrouping is based on primary tumor characteristics, ulceration of the primary tumor, loco-regional spreading, and number of affected lymph nodes (4). This impaired prognosis is despite the initial curative attempt of radical surgery to remove the primary tumor and loco-regional metastases (4).

Based on the high risk of relapse of stage III melanoma patients, which by surgical interventions are rendered to no evidence of disease (NED), adjuvant therapy would be indicated. The only currently approved adjuvant therapy - despite a recently reported positive clinical trial for adjuvant ipilimumab therapy of stage III melanoma - is interferon- $\alpha$ (IFN $\alpha$ ). In 1995, high-dose IFN $\alpha$ $2 \mathrm{~b}$, and in 2011, pegylated IFN $\alpha-2 b$ has been approved by the FDA for melanoma patients who are at high risk of recurrence. However, the percentage of patients indeed benefiting from adjuvant IFN $\alpha$ therapy is limited. Consequently, ever since the first reports demonstrating anti-tumor effects of IFN $\alpha$ for melanoma (5), several studies have been conducted to identify the optimal therapeutic schedule and the benefiting patient subpopulation. Meta-analyses of these trials demonstrated that IFN $\alpha$ has a 
consistent effect on relapse-free survival but no or only marginal effect on overall survival (OS), which had been attributed to the fact that only a subpopulation of patients benefit from treatment. A recent meta-analysis of two EORTC trials confirmed that limited tumor burden in stage III and ulceration of the primary tumor were the only predictive factors for adjuvant IFN $\alpha$ therapy (6).

Recent observations in other cancers suggest that the efficacy of IFN $\alpha$ therapy depends on the genetic predisposition. A screen of 463 single nucleotide polymorphisms (SNPs) in 33 candidate genes in metastatic renal cell carcinoma patients receiving IFN $\alpha$ therapy demonstrated a significant association of the signal transducer and activator of transcription 3 (STAT3) SNP rs4796793 with clinical response (7). STAT3 is an integral molecule of the IFN $\alpha$ receptor signaling (8) and the rs4796793 SNP correlated with STAT3 mRNA expression (7). This SNP is located in the $5^{\prime}$ region of the gene, $1633 \mathrm{bp}$ upstream of the ATG site. In addition, in a murine melanoma model, blockade of STAT3 enhanced the therapeutic efficacy of IFN-alpha immunotherapy (9). These observations prompted us to scrutinize the impact of rs4796793 on the therapeutic efficacy of adjuvant IFN $\alpha$ in melanoma. Here, we report that despite the fact that there was no correlation between STAT3 mRNA expression and genotype, melanoma cells carrying the minor allele were more sensitive to IFN $\alpha$ in vitro. However, this notion did not translate into the clinical situation as the STAT3 rs4796793 genotype did not correlate with the outcome of adjuvant IFN $\alpha$ treatment in stage III melanoma.

\section{PATIENTS AND METHODS GENOTYPING}

TaqMan allelic discrimination assay for SNP rs4796793 genotyping was purchased from Applied Biosystems (C27977213; Foster City, CA, USA). Polymerase chain reaction (PCR) was performed according to the manufactures instructions in $20 \mu \mathrm{l}$ volume reactions with $1 \mu \mathrm{l}$ DNA on a 7500 Fast Real time PCR system (Applied Biosystems).

\section{QUANTITATIVE RT-PCR ANALYSES FOR STAT3}

Endogeneous STAT3 levels were determined for 35 peripheral blood lymphocytes (PBL) samples as well as 18 melanoma cell lines by real time PCR analyses in TaqMan technology using the comparative $\Delta \Delta C_{\mathrm{T}}$ method. PBL samples were obtained from melanoma patients who did not receive therapy at the time point the blood was drawn. Total RNA was isolated from approximately $3 \times 10^{6}$ cells. Samples of total RNA were subjected to reverse transcription. Primers and probe for STAT3 were designed with Primer Express software (Applied Biosystems, Weiterstadt, Germany). The assay (sense 5'-GGG CAC AAA CAC AAA AGT GAT G; antisense 5'-CAG CTC CTC AGT CAC AAT CAG G; probe 5'-FAM-AGA ATT CAA ACA CTT GAC CCT GAG GGA GCA) detects all three STAT3 mRNA transcript variants. GAPDH (Applied Biosystems) served as endogenous control. The relative expression levels of STAT3 normalized to GAPDH and relative to the PBL sample pat1 heterozygote for the SNP was calculated as $2^{\Delta \Delta C T}$ with $\Delta \Delta C_{\mathrm{T}}=\left(C_{\mathrm{T} \text { STAT3, sample }}-C_{\mathrm{T} \text { GAPDH, sample }}\right)-\left(C_{\mathrm{T} \text { STAT3, pat1 }}-\right.$ $C_{\mathrm{T}}$ GAPDH, pat1). $C_{\mathrm{T}}$ is defined as the cycle when the threshold level of fluorescence is reached.

\section{CELL CULTURE}

Eleven melanoma cell lines were cultured in RPMI 1640 medium supplemented with $10 \%$ fetal calf serum. Four of these had the CC genotype (BLM, M19, M26, MelJuso), three the both genotype (FM79, FM82, Mel2A), and the remaining four the GG genotype (SkMel28, MaMel60, MaMel71, Mel888).

\section{MTS ASSAY}

In order to determine the impact of IFN $\alpha$ on melanoma cells, the MTS [3-(4,5-dimethylthiazol-2-yl)-5-(3-carboxymethoxyphenyl)2-(4 sulfophenyl)-2H-tetrazolium] cell proliferation assay (Promega) was used according to the manufactures instructions. MTS is a tetrazolium reagent that is reduced by metabolically active cells. Melanoma cell lines were cultivated in triplicates in 96 well plates at 1000 (BLM, FM82, M26, Mel2A, Mel888, MelJuso, SKmel28), 4000 (FM79, M19, MaMel60), or 8000 (MaMel71) cells per well with normal medium or supplemented with $51,200 \mathrm{U} / \mathrm{ml}$ IFN $\alpha$ for 4 days. Extinction at $490 \mathrm{~nm}$ and background at $650 \mathrm{~nm}$ were measured with the Spectrostar ${ }^{\text {Nano }}$ (BMG Labtech, Ortenberg, Germany). First, with the blank corrected extinctions, the growth of the cells compared to the basal metabolic rate determined on day 1 before addition of IFN $\alpha$ was calculated. The inhibitory effect of IFN was then determined by $\left(\right.$ growth $_{\text {medium control }}-$ growth $\left._{\text {IFN } \alpha}\right) /$ growth $_{\text {medium control }}{ }^{\star} 100$.

\section{PATIENTS}

Serum from advanced melanoma patients from frozen serum banks hosted by Skin Cancer Unit, Mannheim and the Department of Dermatology, Würzburg served as DNA source for genotyping as previously described (10). In order to be included into the study, the following criteria had to be fulfilled: (i) patients with histologically confirmed melanoma, (ii) a stage III diagnosis with a minimum follow-up of 2 months, (iii) Caucasian origin, and (iv) extended information available on their medical history including whether patients received adjuvant IFN therapy after stage III diagnosis. Patients with secondary malignancies were excluded from the study. Detailed patient characteristics are given in Table 1 . The collection of sera and clinical data were performed after patients' informed consent with Institutional Review Board approval. The presented work was conducted according to the principles expressed in the Declaration of Helsinki.

\section{STATISTICAL METHODS}

Statistical analysis was performed with Prism (GraphPad La Jolla, CA, USA) or Stata 11.2 (StataCorp LP, College Station, TX, USA). For univariate analyses, the Kaplan-Meier method was used to compare survival time between groups. Differences of survival time were assessed by the log-rank test. IFN $\alpha$ sensitivity or STAT3 expression of the different groups were compared by one-way ANOVA parametric when the data passed normality testing or else non-parametric, i.e., Kruskal-Wallis with Dunn's post tests. Univariate as well as multivariate analyses Cox's proportionalhazard regression model were applied when the models had passed the proportional-hazard assumption based on Schoenfeld residuals. 
Table 1 | Patient characteristics

\begin{tabular}{|c|c|c|c|}
\hline & All & $\begin{array}{c}\text { No adjuvant } \\
\text { therapy }\end{array}$ & $\begin{array}{l}\text { Adjuvant IFN } \alpha \\
\text { therapy }\end{array}$ \\
\hline \multicolumn{4}{|l|}{ Gender } \\
\hline F & 119 (46.0\%) & $82(44.8 \%)$ & $37(48.7 \%)$ \\
\hline M & $140(54.1 \%)$ & $101(55.2 \%)$ & $39(51.3 \%)$ \\
\hline $\begin{array}{l}\text { Median age at } \\
\text { diagnosis [IQR] }\end{array}$ & $54.3[41.4-64.7]$ & 56.7 [43-65.5] & 48.9 [35.9-61.6] \\
\hline \multicolumn{4}{|c|}{ Histological type } \\
\hline ALM & $26(10.0 \%)$ & $20(10.9 \%)$ & $6(7.9 \%)$ \\
\hline LMM & $5(1.9 \%)$ & $5(2.7 \%)$ & 0 \\
\hline NM & $94(36.3 \%)$ & $66(36.1 \%)$ & $28(36.8 \%)$ \\
\hline SSM & $70(27.0 \%)$ & $49(26.8 \%)$ & $21(27.6 \%)$ \\
\hline Other & $7(2.7 \%)$ & $5(2.7 \%)$ & $2(2.6 \%)$ \\
\hline Non-classifiable & $7(2.7 \%)$ & $7(3.8 \%)$ & 0 \\
\hline Unknown & $50(19.3 \%)$ & $31(16.9 \%)$ & $19(25.0 \%)$ \\
\hline \multicolumn{4}{|l|}{ Ulceration pT } \\
\hline yes & $57(22.0 \%)$ & $43(23.5 \%)$ & $14(18.4 \%)$ \\
\hline no & $109(42.1 \%)$ & $80(43.7 \%)$ & $29(38.2 \%)$ \\
\hline unknown & $93(35.9 \%)$ & $60(32.8 \%)$ & $33(43.4 \%)$ \\
\hline \multicolumn{4}{|c|}{ STAT3 rs4796793 } \\
\hline $\mathrm{CC}$ & $135(52.1 \%)$ & $99(54.1 \%)$ & $36(47.4 \%)$ \\
\hline Both & $102(39.4 \%)$ & $68(37.2 \%)$ & $34(44.7 \%)$ \\
\hline GG & $22(8.5 \%)$ & $16(8.7 \%)$ & $6(7.9 \%)$ \\
\hline
\end{tabular}

ALM, acral-lentiginuous melanoma; LMM, lentigo maligna melanoma; IQR, interquartile range; NM, nodular melanoma; SSM, superficial spreading melanoma.

\section{RESULTS}

\section{STAT3 rs4796793 GENOTYPE'S IMPACT ON STAT3 mRNA EXPRESSION AND IFN $\alpha$ SENSITIVITY}

It has been previously reported that the STAT3 rs4796793 genotype correlates with endogenous STAT3 expression in lymphocytes (7). To test the relevance of this observation in melanoma, particular in melanoma patients, we genotyped PBL and melanoma cell lines for STAT3 rs4796793 SNP and subsequently measured the STAT3 mRNA expression these. As expected from the role of STAT3 for lymphocytes, its expression was significantly higher in PBLs than in the melanoma cell lines ( $p<0.0001$; Kruskal-Wallis). However, within the two cell types, we could not detect a relevant difference of expression based on the SNP genotype (Figure 1A). Nevertheless, we next established if the STAT3 rs4796793 was associated with IFN $\alpha$ sensitivity of melanoma cell lines. This analysis revealed a clear trend toward an increased IFN $\alpha$ sensitivity of melanoma cell lines with a homozygote STAT3 rs4796793 minor allele. Indeed, the IFN $\alpha$ sensitivity increased from homozygote major allele to heterozygote and to homozygote with minor allele. This difference, however, was statistically not significant $(p=0.1259$, Kruskal-Wallis; Figure 1B).

\section{STAT3 rs4796793 GENOTYPE'S IMPACT ON THE CLINICAL COURSE OF MELANOMA}

Two patient cohorts, i.e., with or without adjuvant IFN $\alpha$ therapy, were included to be able to distinguish if STAT3 rs4796793 SNP is a predictive or a mere prognostic biomarker. Of the 259 patients, who were included, all had been diagnosed with or progressed to stage III melanoma. One hundred nineteen were female (46\%) and 140 male $(54.1 \%)$. The median age at diagnosis of stage III was 56.5 years. The median follow-up time from stage III diagnosis was 38.9 months; within this follow-up time, 159 patients developed distant metastases and 136 deaths were observed. About one-third $(n=76)$ of the patients had received IFN $\alpha$ as adjuvant therapy in stage III. Follow-up times for patients with or without adjuvant IFN $\alpha$ therapy were similar, but the treated cohort was significantly younger both at initial diagnosis or at progression to stage III $(p=0.008)$. Information on presence of absence of ulceration was available for $64.1 \%$ of the patients, i.e., $22 \%$ with, $42.1 \%$ without, and $35.9 \%$ with unknown ulceration status; these subpopulations were equally distributed among the two cohorts. Detailed patient and tumor characteristics and the genotypic frequencies of the STAT3 rs4796793 SNP are given in Table 1. From all patients, the genotype could be determined. The observed genotype frequencies are similar to the frequencies reported for Europeans on the SNP database websites of the National Center for Biotechnology Information (dbSNP, http://www.ncbi.nlm.nih.gov/snp/) ranging from 54.2 to $58.3 \%$ for CC, 33.6 to $39 \%$ for CG, and 3.3 to $8.8 \%$ for GG.

Since therapeutic efficacy of adjuvant IFN $\alpha$ therapy is most evident in delay of disease progression, we analyzed the impact of rs4796793 on distant metastasis free survival (DMFS). For both the no-adjuvant-therapy group as well as the IFN $\alpha$-adjuvanttherapy group, Kaplan-Meier analyses did not reveal any significant association between STAT3 rs4796793 SNP genotype and DMFS (Figures 2A,B; $p=0.2053$ or $p=0.9423$, respectively; logrank test). Despite the observation that the SNP genotype had no influence on DMFS in our patient groups, we tested for a potential effect of the STAT3 rs4796793 SNP genotype on OS by the Kaplan-Meier method. Again, the rs4796793 genotype had no impact on survival for patients receiving IFN $\alpha$ adjuvant therapy or not $(p=0.8403$ or $p=0.7061$, respectively; log-rank test; Figures 2C,D). It should be further noticed that when we performed multivariate Cox regression analyses to adjust for gender and age at diagnosis of stage III, the SNP genotype still was not associated with the risk of progression. This was also the case when ulceration of the primary tumor was included in the analyses, but this information was available only for $64.1 \%$ of our patients reducing the size of the patient groups for analysis accordingly (data not shown).

\section{DISCUSSION}

Melanoma is regarded as one of the most lethal skin cancers. This is still true, despite major breakthroughs in melanoma research resulting in new therapies, such as small molecule kinase inhibitors or immune checkpoint blocking antibodies, which have been proven to be both effective and beneficial in advanced melanoma patients (11). Nevertheless, most patients responding to kinase inhibitors develop resistance to these and at best half of the patients respond to checkpoint blocking antibodies; thus, there is still an indication for adjuvant therapy for high-risk melanoma patients to avoid progression to metastatic disease. The only approved adjuvant therapeutic to date is IFN $\alpha$. Unfortunately, a recent metaanalysis of 14 randomized clinical trials could neither identify 

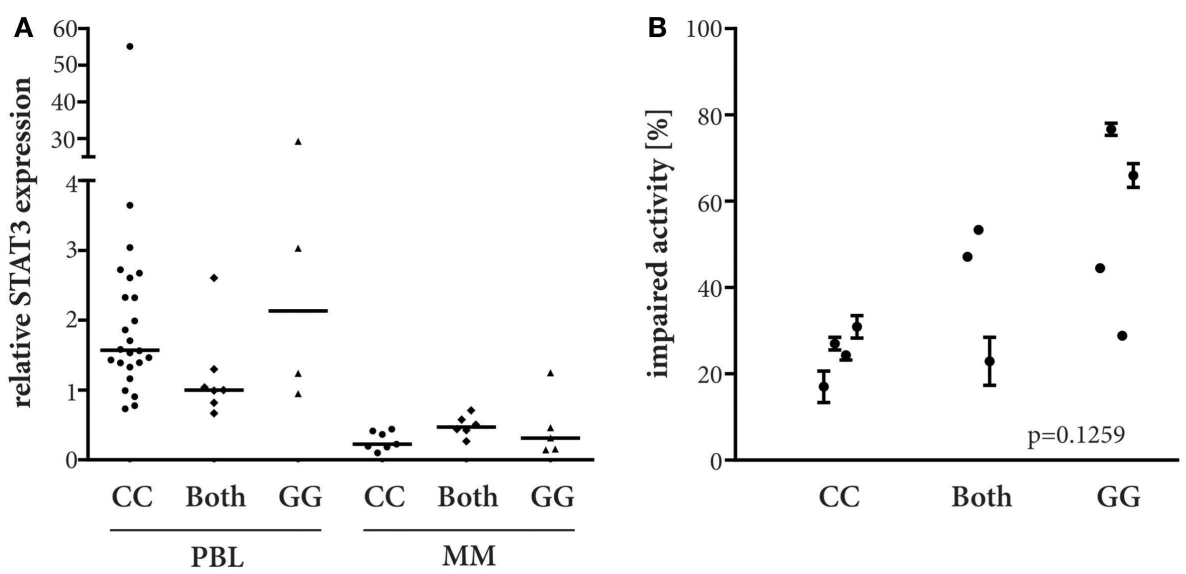

FIGURE 1 | STAT3 rs4796793 dependent endogeneous STAT3 mRNA expression and IFN $\alpha$ sensitivity. (A) STAT3 mRNA expression was measured by real-time PCR in peripheral blood lymphocytes (PBL) and melanoma cell lines (MM). A CG genotype PBL sample served as calibrator. ANOVA analyses did not show significant differences (PBL, $p=0.0863$, Kruskal-Wallis; MM, $p=0.3127$, parametric). (B) MM lines were subjected to $51200 \mathrm{IU} \mathrm{IFN} \alpha / \mathrm{ml}$ or not, after 4 days their metabolic activity was measured by MTS assay. Depicted are the means with standard error for each cell line measured in at least two independent experiments.
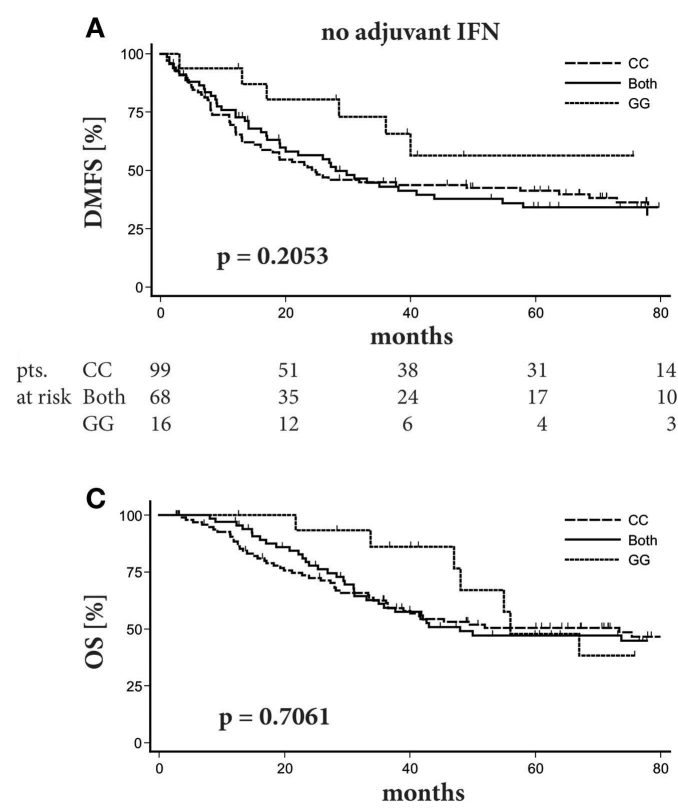

$\begin{array}{llllllr}\text { pts. } & \text { CC } & 99 & 70 & 48 & 37 & 20 \\ \text { at risk } & \text { Both } & 68 & 53 & 24 & 24 & 16 \\ & \text { GG } & 16 & 15 & 10 & 10 & 3\end{array}$

FIGURE 2 | STAT3 SNP rs4796793 does not influence distant metastasis free survival (DMFS) nor overall survival (OS) in stage III melanoma patients. Patients were stratified according to their rs4796793 SNP.
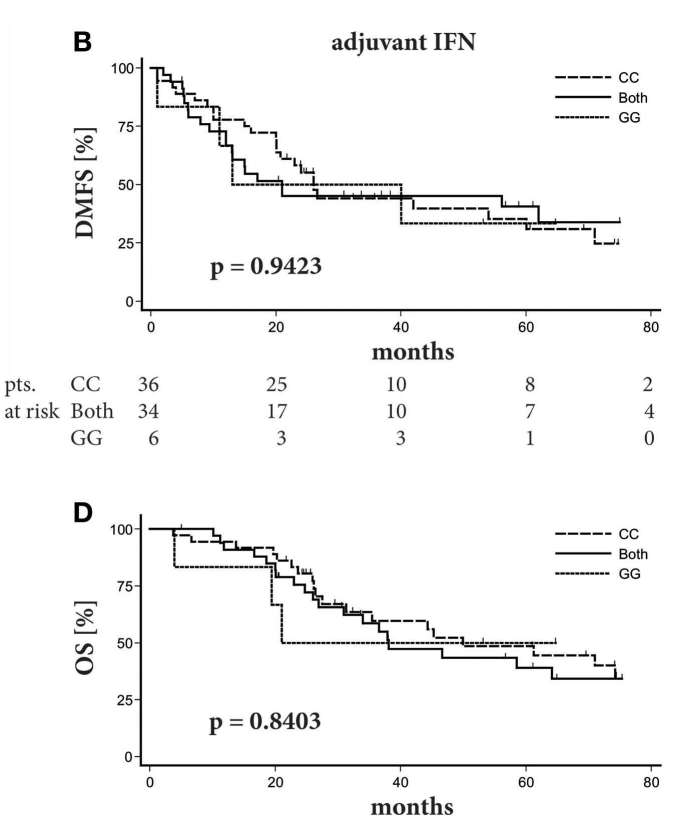

$\begin{array}{llrrrrr}\text { pts. } & \text { CC } & 36 & 32 & 16 & 13 & 6 \\ \text { at risk } & \text { Both } & 34 & 27 & 12 & 9 & 5 \\ & \text { GG } & 6 & 4 & 3 & 2 & 1\end{array}$

Kaplan-Meier plots for DMFS or OS of melanoma patients without $(\mathbf{A}, \mathbf{C})$ or with adjuvant IFN $\alpha$ therapy (B,D) in stage III. Below each graph, the patient numbers (pts.) at risk are given. Log-rank test was performed for statistical analysis. an optimal IFN $\alpha$ dose and/or treatment duration nor the subset of patients benefiting from therapy (12). The latter is of particular importance as adjuvant IFN $\alpha$ therapy is associated with substantial toxicity in most of the patients (13). Consequently, a predictive biomarker would improve the risk (=toxicity) to benefit (=reduced risk of relapse/progression) ratio. In this regard, a recent meta-analysis of two EORTC trials demonstrated that only ulceration of the primary tumor and tumor stage are possible predictive factors for adjuvant IFN $\alpha$ (6).

Clinical efficacy of a therapeutic intervention, however, does not only dependent on tumor characteristics, but is also influenced by the patients' genetics, which is particularly true for immune 
modulating therapies, such as IFN $\alpha$ (14). Notably, the effects of type I IFNs on the adaptive immune system are tightly regulated (15). One of the integral molecules of the IFN receptor signaling is STAT3 (8) and a SNP in STAT3 (i.e., rs4796793) has been reported to be associated with response to IFN $\alpha$ therapy in renal cell carcinoma patients (7). SNPs associated with response to therapy may affect the response either directly or indirectly by being in linkage disequilibrium with other disease-modulating alleles. In the case of rs4796793 and the effect of IFN $\alpha$ in renal cell carcinoma, it is assumed to be a direct effect, since the authors reported a small $\left(R^{2}=0.14\right)$ but significant correlation between rs4796793 and STAT3 mRNA expression in Epstein-Barr virus transformed B-lymphocyte cell lines, i.e., a higher expression in cells being homozygote for the major allele (7). The increased endogeneous expression of STAT3 mRNA in cell lines harboring the major allele of rs4796793 was explained by increased binding of NKX2-5 to the STAT3 promoter, since this genotype contains an additional NKX2-5 binding site. Here, however, we did not observe a significant difference in endogeneous STAT3 mRNA for PBLs or for melanoma cell lines depending on the STAT3 rs4796793 genotype. These cell types may lack the respective transcription factor network, i.e., NKX2-5 and the antagonizing transcription factors NR2F1 and HMX1, or other transcription factors might be more relevant for endogenous STAT3 expression in these cells.

Originally described for their antiviral activity, IFN $\alpha$ subtypes have demonstrated anti-tumor activity in different cancers (16). The therapeutic effect can be subdivided into those directly affecting tumor cells and those, which require immune mechanisms. IFN $\alpha$ exerts multiple biological effects, such as induction of apoptosis and inhibition of cell growth (17). The ability to enhance immune recognition of tumor cells by increasing MHC class I molecule expression resembles the intersection between direct and indirect effects of IFN $\alpha$. The latter comprises several effects on immune competent host cells, e.g., enhancing differentiation of Th1 T-cell responses, generation, and activation of cytotoxic T-cells, as well as differentiation of DCs (16). Preclinical studies suggest that the applied dose determines which anti-tumor effect is triggered (18). In the adjuvant setting, direct effects on tumor cells are difficult - if not impossible - to determine, but the immune modulatory functions of IFN $\alpha$ are clearly evident. For example, in melanoma patients, the appearance of autoantibodies or clinical manifestations of autoimmunity during therapy was associated with an improved DMFS (19). Similarly, a therapyassociated increase of STAT1 activation in PBLs also correlated with the clinical benefit (20).

It is well established that IFN mediates its effect by STAT signaling (16). For example, the interferon-stimulated response elements (ISRE) of interferon-stimulated genes bind protein complexes containing phosphorylated STAT1 and STAT2 (21). Furthermore, it has been demonstrated that STAT3 expression increased the sensitivity to type I interferons in otherwise resistant cell lines (8). STAT3 has also been implicated in the clinical outcome of IFN $\alpha$ therapy. In a small study with 24 patients, the effect of IFN $\alpha$ treatment on phosphorylation of tyrosine at position 705 of STAT3 was determined (22). Phosphorylation of tyrosine 705 is necessary for STAT3 dimerization through phosphotyrosine-SH2 domain, which is a prerequisite for STAT3 transcriptional activity [reviewed in Ref. (23)]. Notably, STAT3 tyrosine 705 phosphorylation status in response to IFN $\alpha$ administration correlated with DMFS and OS. Since type I IFNs are weak activators of STAT3 (24), IFN $\alpha$ sensitivity should already be affected by the STAT3 expression level. Indeed, knockdown of STAT3 enhances IFN $\alpha$ mediated cell growth inhibition in vitro. These observations suggest that direct effect of the STAT3 rs4796793 SNP are responsible for the reported effects on efficacy of IFN $\alpha$ in renal cell carcinoma patients (7). In a recent report by Kreil et al., the association between the STAT5B rs6503691 SNP and response of chronic myeloid leukemia (CML) to IFN $\alpha$ was demonstrated (25). Interestingly, this SNP was not related to STAT5A or STAT5B mRNA expression, but to STAT3 mRNA expression, suggesting an association of STAT3 expression and IFN $\alpha$ efficacy.

Although we did not detect a correlation between the STAT3 rs4796793 genotype and STAT3 mRNA levels, our in vitro results suggest that the presence of the minor allele increases the sensitivity of melanoma cells toward IFN $\alpha$. Nevertheless, this in vitro effect did not translate into a clinical association. In the tested melanoma cohort receiving adjuvant IFN $\alpha$ therapy, no correlation between the STAT3 rs4796793 genotype and DMFS or OS was evident. It should be noted that given the number of patients and the event rate in the IFN $\alpha$ treated patients, a Cox regression for DMFS achieves $80 \%$ power at a 0.05 significance level to detect a hazard ratio of 1.51 . Thus, it is rather unlikely that if the STAT3 rs4796793 SNP has a relevant impact, it would have been missed because of the size of the analyzed cohort. Consequently, the here presented data do not support a significant impact of the STAT3 rs4796793 SNP on IFN $\alpha$ efficacy in melanoma patients.

\section{ACKNOWLEDGMENTS}

We thank Eva-Maria Sarosi, Michelle Holterhus, and Andrea Roschger for excellent technical assistance. This work was supported by the Wilhelm Sander Stiftung [grant \#2006.054.1].

\section{REFERENCES}

1. Simard EP, Ward EM, Siegel R, Jemal A. Cancers with increasing incidence trends in the United States: 1999 through 2008. CA Cancer J Clin (2012) 62(2):118-28. doi:10.3322/caac. 20141

2. Jemal A, Siegel R, Xu J, Ward E. Cancer statistics, 2010. CA Cancer J Clin (2010) 60:277-300. doi:10.3322/caac.20073

3. Ekwueme DU, Guy GP Jr, Li C, Rim SH, Parelkar P, Chen SC. The health burden and economic costs of cutaneous melanoma mortality by race/ethnicity-United States, 2000 to 2006. J Am Acad Dermatol (2011) 65:S133-43. doi:10.1016/j.jaad. 2011.04.036

4. Balch CM, Gershenwald JE, Soong SJ, Thompson JF, Atkins MB, Byrd DR, et al. Final version of 2009 AJCC melanoma staging and classification. J Clin Oncol (2009) 27:6199-206. doi:10.1200/JCO.2009.23.4799

5. Sherwin SA, Knost JA, Fein S, Abrams PG, Foon KA, Ochs JJ, et al. A multipledose phase I trial of recombinant leukocyte A interferon in cancer patients. JAMA (1982) 248:2461-6. doi:10.1001/jama.1982.03330190025025

6. Eggermont AM, Robert C. New drugs in melanoma: it's a whole new world. Eur J Cancer (2011) 47:2150-7. doi:10.1016/j.ejca.2011.06.052

7. Ito N, Eto M, Nakamura E, Takahashi A, Tsukamoto T, Toma H, et al. STAT3 polymorphism predicts interferon-alfa response in patients with metastatic renal cell carcinoma. J Clin Oncol (2007) 25:2785-91. doi:10.1200/JCO.2006.09.8897

8. Yang $\mathrm{CH}$, Murti A, Pfeffer LM. STAT3 complements defects in an interferonresistant cell line: evidence for an essential role for STAT3 in interferon signaling and biological activities. Proc Natl Acad Sci U S A (1998) 95:5568-72. doi:10.1073/pnas.95.10.5568

9. Kong LY, Gelbard A, Wei J, Reina-Ortiz C, Wang Y, Yang EC, et al. Inhibition of p-STAT3 enhances IFN-alpha efficacy against metastatic melanoma in a murine 
model. Clin Cancer Res (2010) 16:2550-61. doi:10.1158/1078-0432.CCR-100279

10. Schrama D, Scherer D, Schneider M, Zapatka M, Brocker EB, Schadendorf D, et al. ERCC5 p.Asp1104His and ERCC2 p.Lys751Gln polymorphisms are independent prognostic factors for the clinical course of melanoma. Invest Dermatol (2011) 131:1280-90. doi:10.1038/jid.2011.35

11. Espinosa E, Berrocal A, Lopez Martin JA, Gonzalez CM, Cerezuela P, Mayordomo JI, et al. Advances in cutaneous melanoma. Clin Transl Oncol (2012) 14:325-32. doi:10.1007/s12094-012-0804-4

12. Mocellin S, Pasquali S, Rossi CR, Nitti D. Interferon alpha adjuvant therapy in patients with high-risk melanoma: a systematic review and meta-analysis. J Natl Cancer Inst (2010) 102:493-501. doi:10.1093/jnci/djq009

13. Ascierto PA, Chiarion-Sileni V, Muggiano A, Mandala M, Pimpinelli N, Vecchio $\mathrm{MD}$, et al. Interferon alpha for the adjuvant treatment of melanoma: review of international literature and practical recommendations from an expert panel on the use of interferon. J Chemother (2014) 26:193-201. doi:10.1179/ 1973947813Y.0000000154

14. Huang RS, Johnatty SE, Gamazon ER, Im HK, Ziliak D, Duan S, et al. Platinum sensitivity-related germline polymorphism discovered via a cell-based approach and analysis of its association with outcome in ovarian cancer patients. Clin Cancer Res (2011) 17:5490-500. doi:10.1158/1078-0432.CCR-11-0724

15. Tough DF. Modulation of T-cell function by type I interferon. Immunol Cell Biol (2012) 90:492-7. doi:10.1038/icb.2012.7

16. Sondak VK, Becker JC, Hauschild A. Interferons. 9th ed. In: DeVita VT, Lawrence TS, Rosenberg SA, editors. DeVita, Hellman, and Rosenberg's Cancer: Principles \& Practice of Oncology. Philadelphia, PA: Lippincott Williams \& Wilkins (2011). p. 461-8.

17. Rizza P, Moretti F, Belardelli F. Recent advances on the immunomodulatory effects of IFN-alpha: implications for cancer immunotherapy and autoimmunity. Autoimmunity (2010) 43:204-9. doi:10.3109/08916930903510880

18. Bracarda S, Eggermont AM, Samuelsson J. Redefining the role of interferon in the treatment of malignant diseases. Eur J Cancer (2010) 46:284-97. doi:10.1016/j.ejca.2009.10.013

19. Gogas H, Ioannovich J, Dafni U, Stavropoulou-Giokas C, Frangia K, Tsoutsos D, et al. Prognostic significance of autoimmunity during treatment of melanoma with interferon. N Engl J Med (2006) 354:709-18. doi:10.1056/ NEJMoa053007

20. Simons DL, Lee G, Kirkwood JM, Lee PP. Interferon signaling patterns in peripheral blood lymphocytes may predict clinical outcome after high-dose interferon therapy in melanoma patients. J Transl Med (2011) 9:52. doi:10.1186/14795876-9-52

21. Darnell JE Jr, Kerr IM, Stark GR. Jak-STAT pathways and transcriptional activation in response to IFNs and other extracellular signaling proteins. Science (1994) 264:1415-21. doi:10.1126/science.8197455

22. Humpolikova-Adamkova L, Kovarik J, Dusek L, Lauerova L, Boudny V, Fait V, et al. Interferon-alpha treatment may negatively influence disease progression in melanoma patients by hyperactivation of STAT3 protein. Eur J Cancer (2009) 45:1315-23. doi:10.1016/j.ejca.2009.01.009

23. Santos CI, Costa-Pereira AP. Signal transducers and activators of transcriptionfrom cytokine signalling to cancer biology. Biochim Biophys Acta (2011) 1816:38-49. doi:10.1016/j.bbcan.2011.03.003

24. Calo V, Migliavacca M, Bazan V, Macaluso M, Buscemi M, Gebbia N, et al. STAT proteins: from normal control of cellular events to tumorigenesis. J Cell Physiol (2003) 197:157-68. doi:10.1002/jcp.10364

25. Kreil S, Waghorn K, Ernst T, Chase A, White H, Hehlmann R, et al. A polymorphism associated with STAT3 expression and response of chronic myeloid leukemia to interferon alpha. Haematologica (2010) 95:148-52. doi:10.3324/ haematol.2009.011510

Conflict of Interest Statement: The authors declare that the research was conducted in the absence of any commercial or financial relationships that could be construed as a potential conflict of interest.

Received: 08 September 2014; accepted: 12 November 2014; published online: 28 November 2014.

Citation: Schrama D, Ugurel S, Sucker A, Ritter C, Zapatka M, Schadendorf D and Becker JC (2014) STAT3 single nucleotide polymorphism rs4796793 SNP does not correlate with response to adjuvant IFN $\alpha$ therapy in stage III melanoma patients. Front. Med. 1:47. doi: 10.3389/fmed.2014.00047

This article was submitted to Dermatology, a section of the journal Frontiers in Medicine.

Copyright (C) 2014 Schrama, Ugurel, Sucker, Ritter, Zapatka, Schadendorf and Becker. This is an open-access article distributed under the terms of the Creative Commons Attribution License (CC BY). The use, distribution or reproduction in other forums is permitted, provided the original author(s) or licensor are credited and that the original publication in this journal is cited, in accordance with accepted academic practice. No use, distribution or reproduction is permitted which does not comply with these terms. 